\title{
Market calibration under a long memory stochastic volatility model
}

\author{
Tomáš Sobotka ${ }^{1}$
}

\section{Introduction}

We study a stochastic volatility model of financial asset dynamics firstly proposed by Intarasit and Sattayatham (2011). Under the model, stock prices follow a jump-diffusion stochastic process and its stochastic volatility is driven by a continuous-time fractional process that attains a long memory. Unlike many other "classical" models of market dynamics this approach should take into account most of the observed market aspects as volatility clustering, leverage effect and pronounced skewness of the volatility smile.

The reason why many practitioners tend to use simpler and less realistic modelling approaches is due to the common knowledge that the more complex model one employs, more restrictions one has to impose in order to use the model on practical tasks. The fractional stochastic volatility model introduced in the following section, however, stays tractable and is convenient for various practical tasks which will be illustrated on calibration from European option markets.

\section{Model and its option pricing formula}

We consider a market with a risk-free and risky asset. One assumes that a price of the former asset is compounded using a constant (and known) risk-free rate $r$ continuously in time. As the latter asset one might consider e.g. stocks, commodities or indexes. According to the FSV approach, the risky asset is modelled by stochastic differential equations (1)-(2) which under the risk-neutral measure take the following form

$$
\begin{aligned}
d S(t) & =r S_{t} d t+\sqrt{v(t)} S(t) d W(t)+Y(t) S(t-) d N(t) \\
d v(t) & =-\kappa[v(t)-\bar{v}] d t+\xi v(t) d B(t) \\
S(0) & =S_{0} ; \quad v(0)=v_{0}
\end{aligned}
$$

where $\kappa, \bar{v}, \xi$ are model parameters, such that, $\kappa$ is a mean-reversion rate, $\bar{v}$ stands for an average volatility level and finally, $\xi$ is so-called volatility of volatility. Under the notation $S_{t-}$ we understand $\lim _{\tau \rightarrow t-} S_{\tau}$ and $N(t)_{t \in \mathbb{R}^{+}}, W(t)_{t \in \mathbb{R}^{+}}$is a Poisson process and a standard Wiener process respectively. $B(t)$ is a fractional process with Hurst parameter $H \in(0,1)$. The process has a long memory (unlike Wiener process) for $H>1 / 2$. Thus we consider only values of the Hurst parameter in this range. More information on the process and corresponding stochastic differential $d B(t)$ can be found in Pospíšil and Sobotka (2015); Thao (2006).

Under the aforementioned market dynamics and standard assumptions, one can compute a fair price of a contract that pays $(S(T)-K)^{+}$at future time $T$ where $K$ is a known constant

\footnotetext{
${ }^{1}$ student of the doctoral study programme Mathematics, field Applied Mathematics, e-mail: sobotkat@ntis.zcu.cz
} 
(Strike price). This contract is called a European call option and, according to the FSV model, it should take value $C(T, K)$ at time $t<T$,

$$
C(T, K)=S_{t} P_{1}(S(t), v(t), T-t)-e^{-r(T-t)} K P_{2}(S(t), v(t), T-t),
$$

where $P_{1}, P_{2}$ are in the money probabilities that can be retrieved by inverse Fourier transform from a characteristic function of the stock price process. The characteristic function that is used to evaluate $P_{1}, P_{2}$ was derived in Pospíšil and Sobotka (2015).

\section{Real market calibration}

As the price evolution $S(t)$ is a stochastic process, one cannot use the model to predict future prices. Possible applications include evaluation of hedging ratios (i.e. to insure portfolio against a value loss) and pricing over-the-counter financial derivatives. For both of these tasks it is necessary to calibrate model parameters beforehand.

To test calibration performance we employed a data set that consists of 82 call options on FTSE 100 index traded on $8^{\text {th }}$ January 2014. By several optimization techniques we managed to calibrate the FSV model with reasonable error measures, especially compared to a simpler, but very popular Heston (1993) model.

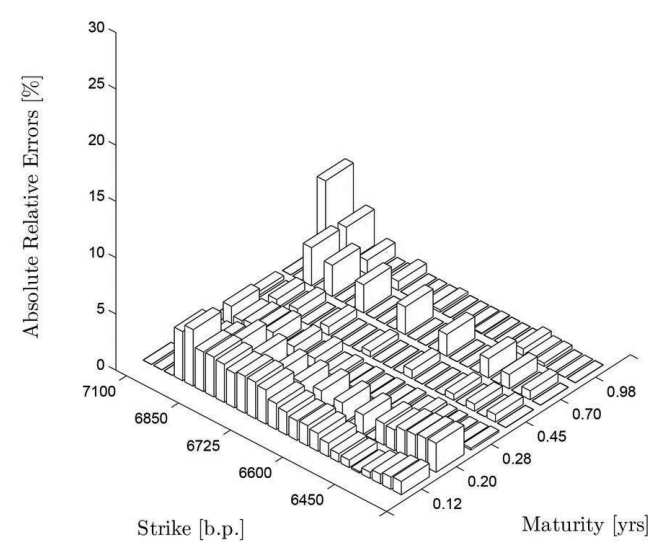

(a) FSV model

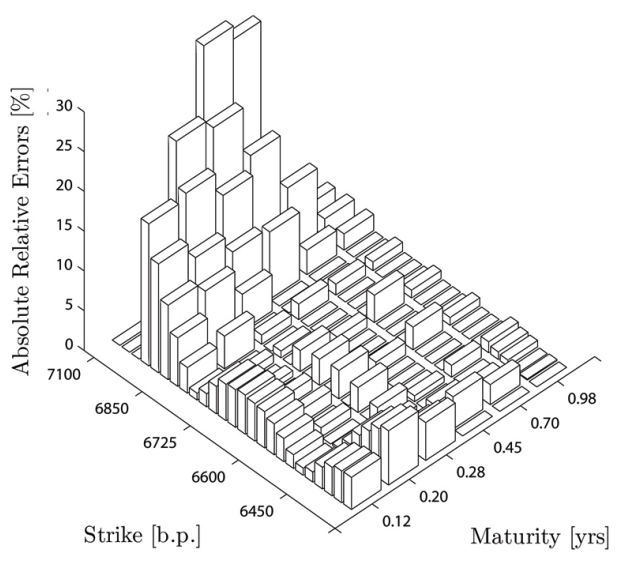

(b) Heston model

\section{Acknowledgement}

The work has been supported by the grant of The University of West Bohemia, project No. SGS-2013-022 and by GA ČR, project No. GA14-11559S.

\section{References}

Heston S. L., 1993. A Closed-Form Solution for Options with Stochastic Volatility with Applications to Bond and Currency Options. Review of Financial Studies, Vol. 6. pp 327-343.

Intarasit, A. and Sattayatham, P., 2011. An Approximate Formula of European Option for Fractional Stochastic Volatility Jump-Diffusion Model. Journal of Mathematics and Statistics, Vol. 7. pp 230-238.

Pospíšil, J. and Sobotka, T., 2015. Market calibration under a long memory stochastic volatility model. [Under Review].

Thao, T. H., 2006. An approximate Approach to fractional analysis for Finance. Nonlinear Analysis: Real world Applications, Vol. 7. pp 124-132. 